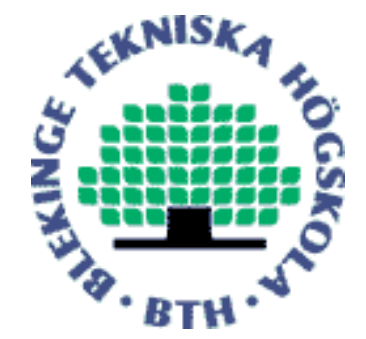

Copyright (C) 2012 IEEE.

Citation for the published paper:

Competition-Based Channel Selection for Cognitive Radio Networks

Yong Yao, Said Rutabayiro Ngoga, David Erman, Adrian Popescu

Wireless Communications and Networking Conference WCNC

2012 Shanghai

This material is posted here with permission of the IEEE. Such permission of the IEEE does not in any way imply IEEE endorsement of any of BTH's products or services Internal or personal use of this material is permitted. However, permission to reprint/republish this material for advertising or promotional purposes or for creating new collective works for resale or redistribution must be obtained from the IEEE by sending a blank email message to pubs-permissions@ieee.org.

By choosing to view this document, you agree to all provisions of the copyright laws protecting it. 


\title{
Competition-Based Channel Selection for Cognitive Radio Networks
}

\author{
Yong Yao, Said Rutabayiro Ngoga, David Erman, and Adrian Popescu \\ School of Computing, Blekinge Institute of Technology, 37179 Karlskrona, Sweden \\ Email: \{yya, srn, der, apo\}@bth.se
}

\begin{abstract}
In cognitive radio networks, unlicensed users need to learn from environmental changes. This is a process that can be done in a cooperative or non-cooperative manner. Due to the competition for channel utilization among unlicensed users, the non-cooperative approach may lead to overcrowding in the available channels. This paper is about a fuzzy logic based decision making algorithm for competition-based channel selection. The underlying decision criterion integrates both the statistics of licensed users' channel occupancy and the competition level of unlicensed users. By using such an algorithm, the unlicensed user competitors can achieve an efficient sharing of the available channels. Simulation results are reported to demonstrate the performance and effectiveness of our suggested algorithm.
\end{abstract}

Index Terms-cognitive radio networks, competition, decision making, fuzzy logic.

\section{INTRODUCTION}

Cognitive Radio (CR) networks is an emerging technology advanced to solve the problem of spectrum scarcity. In CR networks, the licensed spectrum channels are either exclusively reserved for licensed users (i.e., primary users or PUs) or temporarily used by unlicensed users (i.e., secondary users or SUs). Extensive research has been done to develop this concept, based on which the SUs are allowed to access the available channels (also known as spectrum holes) not being occupied by PUs [1]. Moreover, when the PU occupies a channel, the SU in the same channel must leave. Otherwise, the PU transmission would be impaired.

Since PUs do not need to notify SUs of their activities, a time-slotted transmission scheme is suggested for SUs to transmit in CR networks. In this scheme, the SU's transmission is divided into identical slots over time [2]. During each slot, the SU first performs spectrum sensing to detect channel availability. Then the SU may transmit data via an available channel (if it exists) within the remaining slot duration. Further, to alleviate the interruption from PUs, SUs need to learn from the statistical information about PUs' activity, and thus select the most available channels to use. One existing solution along with this line is given by the idle time based statistics. For a single channel, being idle indicates the PU absence and the idle time indicates how long this absence is. In [3], the authors consider that the longer an available channel remains idle in the near future, the higher the channel availability becomes. Further, by predicting the idle time, the most available channel is attributed to the characteristic of having the longest remaining idle time.
In a CR network, the idle time statistics can be shared by multiple SUs. When several SUs simultaneously want to access available channels, the selection criterion based on the longest remaining idle time may lead to the same channels. In particular, the SUs that can perceive (by receiving radio signals) each other are likely to compete for the channel utilization over a single channel. We call these SUs competitors. As the channel capacity is limited, the single channel may not satisfy the requirements of all SU competitors. Further, if a channel is overcrowded due to a large number of SU competitors, the Quality of Service (QoS) performance degrades [4]. Therefore, it is necessary to allow SUs get access to different available channels as much as possible.

In this paper, we jointly consider the idle time and SUs' competition for channel selection. We suggest a Two-Step Information-Exchange (TSIE) method to address a competition problem among SUs in an ad-hoc environment. By applying fuzzy logic, we develop a hybrid decision making algorithm of integrating the idle time statistics and SU competition level. The goal of this algorithm is to provide channel selection with a leverage for long remaining idle time and low-level SU competition.

The rest of the paper is organized as follows. In Section II, we present the system model and related work. Section III discusses the learning of idle time statistics. The competition problem and the suggested TSIE method are described in Section IV. Section V is about the hybrid decision making algorithm. The performance evaluation is presented in Section VI. Finally, we conclude the paper in Section VII.

\section{System MOdEL AND RELATED WORK}

We consider a CR network system with $N$ licensed channels marked with index $1,2, \ldots, N$, respectively. The PUs activity on these channels is assumed to use a synchronous timeslotted basis. Each PU's slot has an uniform length $\delta$ in time domain. In the system, there are $M$ SUs having the labels $s_{1}, s_{2}, \ldots, s_{M}$, respectively. We define $S$ as the set of $M$ SUs, i.e., $S=\left\{s_{1}, s_{2}, \ldots, s_{M}\right\}$. These SUs are ready to transmit data to other SU receivers in a single-hop ad-hoc manner.

We assume that a central coordinator is used in the system. The coordinator can be, e.g., a secondary base station or a support node [5][6]. Similar to [5], the collaborative spectrum sensing is done on both coordinator and SU sides, and thus the probabilities of missed detection and false alarm can be decreased. We therefore assume that the sensing results 
are perfect. The central coordinator is also responsible for realizing PU's slot information, and thus periodically performs sensing with duration $\delta$ corresponding to PU's activity. Both PU's slot information and sensing results are broadcasted by the coordinator to SUs via a Common Control Channel (CCC) [1][6]. Moreover, the coordinator helps every SU transmitter/receiver pair in establishing the reliable communication ${ }^{1}$.

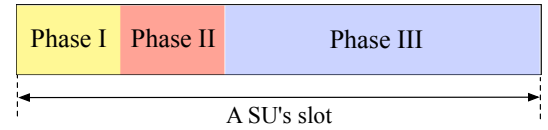

Fig. 1. SU's slot: sensing \& receiving broadcast, information exchange and data transmission are accomplished in the phases I, II and III, respectively.

The SUs use the time-slotted transmission scheme to opportunistically access available channels. By receiving broadcasts from the central controller, the SUs can be synchronized with the PUs ${ }^{2}$. Further, the SU configures its transmission slot length as $\delta$. More specifically, a SU's slot consists of three phases, as shown in Fig 1. In the figure, the sensing and receiving broadcast are accomplished within the first phase. The second and third phases are used for SUs to cooperatively exchange information and to transmit data, respectively. The information exchange among SUs can be accomplished through either the CCC or cooperative mechanisms like, e.g., signaling protocol [7]. For data transmission, in [8] the authors suggest to divide the third phase into multiple identical subslots. Further, by using a modified CSMA/CA protocol, several SU competitors over the same channel can use different subslots to transmit data with low-level collision.

In our work, we assume that the above mentioned functions, i.e., perfect spectrum sensing, coordinator's broadcasting, information exchange, sub-slot and CSMA/CA based transmission, are applicable in the modeled system. However, in the paper we will not deeply study these functions. We instead focus on the joint consideration of idle time statistics and SUs' competition problem.

\section{IDLE TIME StATISTICS}

Unlike [3], we do not assume that SUs have a priori knowledge about distribution parameters regarding idle time like, e.g., the PU arrival and departure rates. Thus, the learning of idle time statistics requires a short-term historical information about PU channel occupancy.

\section{A. PU Channel Occupancy}

Given the current time $t$, the PU's activity may have a change at time points $\{t-H \delta, t-(H-1) \delta \ldots, t\}$. Namely, the time interval $[t-H \delta, t]$ is identically divided into $H$ time slots, within each of which PUs are either present or absent.

\footnotetext{
${ }^{1}$ In [2], the authors suggest a POMDP based method to achieve the channel synchronization on a SU transmitter/receiver pair. However, due to the dynamic nature of both PU's and SU's activities, the design of precise and reliable channel synchronization is very challenging in ad-hoc CR networks.

${ }^{2}$ To differentiate PU's signal from SU's signal, the SUs usually keep silence at the same time while doing sensing [5].
}

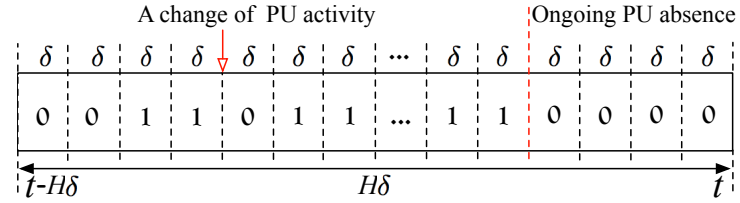

Fig. 2. A binary sequence, which indicates the PU channel occupancy.

We let $h$ denote the slot $[t-(H-h) \delta, t-(H-h-1) \delta]$, where $h=0,1, \ldots, H-1$. Let the random variable $v_{h}^{n}$ denote the sensing result of detecting the PU's activity on channel $n \in\{1,2, \ldots, N\}$ in slot $h$. Then, $v_{h}^{n}$ is specified as $v_{h}^{n} \in\{1$ (PU presence), 0 (PU absence) $\}$. This gives a binary sequence indicating PU channel occupancy. We schematically illustrate the sequence in Fig. 2.

At time $t$, if channel $n$ is sensed to be idle, and we have $v_{H}^{n}=0$. This means that the channel $n$ will be idle in the whole interval $[t, t+\delta]$ and may remain idle in one or more consecutive slots after the time point $(t+\delta)$. As such, the capability of looking ahead the future trends of all channels is desirable for SUs. Namely, we are faced with the task of knowing in advance the remaining idle time on every channel.

\section{B. Remaining Idle Time}

To achieve the above mentioned task, we first compute the average idle time and ongoing PU absence on a channel $n$ (available at time $t$ ) during the past interval $H \delta$.

To compute the average idle time, we are interested in the occurrence times of two events, namely, the event " $v_{h}^{n}=0$ " and the event "PU being absent". We observe in Fig. 2 that the occurrence time of the first event equals $\sum_{h=0}^{H-1}\left(1-v_{h}^{n}\right)$. The occurrence time of the second event is computed with $\left\lfloor\frac{1}{2} \sum_{h=0}^{H-1} \Lambda(n, h) \mid+1\right.$, where $\Lambda(n, h)$ means a change of PU activity and equals 1 if $v_{h}^{n} \neq v_{h+1}^{n}$, otherwise 0 . We then have the average idle time on channel $n$ in interval $[t-H \delta, t]$ as:

$$
E_{\text {idle }}^{n}(t)=\frac{\left\lfloor\frac{1}{2} \sum_{h=0}^{H-1} \Lambda(n, h)\right\rfloor+1}{\sum_{h=0}^{H-1}\left(1-v_{h}^{n}\right)}
$$

Let $x^{n}(t)$ denote the time period of the ongoing PU absence on channel $n$ until time point $t$. For instance, in Fig.2, the last four slots before time $t$ are associated with an ongoing PU absence. To compute $x^{n}(t)$, we find out the slot, in which the latest event " $v_{h}^{n}=1$ " takes place. Let $h^{\prime}$ denote this slot and we have $h^{\prime}=\max \left\{h \mid v_{h}^{n}=1: h=0,1, \ldots, H-1\right\}$. Then, we can obtain $x^{n}(t)=t-H \delta-h^{\prime} \delta$.

Actually, $E_{i d l e}^{n}(t)$ provides an insight into how long the duration of a PU absence is expected to be. In contrast to the remaining idle time described in Section I, the larger $x^{n}(t)$ is, the lower the availability of channel $n$ becomes. In Section $\mathrm{V}$, we will present the fuzzy logic based channel availability estimation with respect to parameters $E_{i d l e}^{n}(t)$ and $x^{n}(t)$.

\section{Competition Problem And Suggested Solution}

For simplicity, we assume that when the SUs (in the modeled system) do single-hop ad-hoc transmission, they have 
the same transmission range $\mathbb{D}$. We let $d_{i j}(t)$ denote at time $t$ the distance between two different SUs, e.g., $s_{i}, s_{j} \in S$, and let $\xi_{i j}(t)$ denote a relation of whether or not $s_{i}$ can perceive $s_{j}$. Then, $\xi_{i j}=1$ if $d_{i j}(t) \leq \mathbb{D}$, and $\xi_{i j}=0$ if $d_{i j}(t)>\mathbb{D}$, where $\xi_{i j}(t)=\xi_{j i}(t)$. When $\xi_{i j}(t)=1, s_{i}$ is said to be a neighbor of $s_{j}$. If both $s_{i}$ and $s_{j}$ switch to the same available channel for data transmission, they become SU competitors against each other.

\section{A. Competition Problem}

To address the competition problem, we first introduce a measure called sub-slot utilization. Consider a slot $h$, in which the transmission phase is identically divided into $L$ sub-slots, each denoted by $1,2, \ldots, L$, respectively. Given a channel $n \in$ $\{1,2, \ldots, N\}$ available in slot $h$, a SU $s_{m} \in S$ attempts to access channel $n$ and to transmit data within the particular sub-slots. Clearly, when $s_{m}$ 's activity follows a CSMA/CAlike protocol model, its transmission may only take place in a subset of all $L$ sub-slots. Let $l_{m}^{n}(h)$ denote the number of used sub-slots in slot $h$, i.e., $0 \leq l_{m}^{n}(h) \leq L$. We thus define the sub-slot utilization, denoted by $u_{m}^{n}(h)$, of $s_{m}$ on channel $n$ in slot $h$ as the ratio between the number of used sub-slots and the number of total sub-slots, i.e., $u_{m}^{n}(h)=l_{m}^{n}(h) / L$.

The reliable communication between SU transmitter and receiver is therefore constrained by the sub-slot utilization threshold, denoted by $\mathbb{U}$. In other words, if $u_{m}^{n}(h)$ is less than $\mathbb{U}$ (due to other SU competitors), $s_{m}$ may terminate transmission since the QoS performance may not be satisfied by the receiver any more.

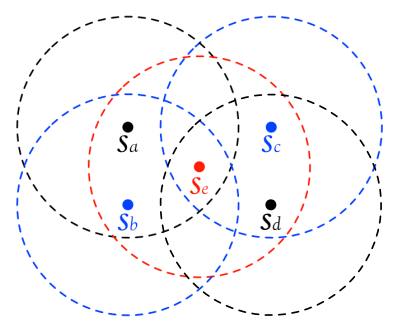

Fig. 3. Example of five SUs competing for the use of the same channel.

We illustrate a competition example in Fig 3. In the figure, five SU transmitters, denoted by $s_{a}, s_{b}, s_{c}, s_{d}, s_{e} \in S$, want to use the same available channel $n$ within slot $h$. Assume a particular threshold $\mathbb{U}=25 \%$. For $s_{a}, s_{b}, s_{c}, s_{d}$, each of them can perceive other two SU transmitters, so that the number of competitors for them is three. Thus, the largest sub-slot utilization allowed for each of them to hold is $50 \%$. However, for $s_{e}$ the number of competitors is five, since $s_{e}$ can perceive four other SU transmitters. As a result, all of $s_{a}, s_{b}, s_{c}, s_{d}$ could successfully use channel $n$ in slot $h$, but $s_{e}$ could not do reliable transmission on channel $n$ in slot $h$ because of not enough sub-slots to use.

As an another example, assume that the channel $n$ was also available in slot $(h-1)$ and SUs $s_{b}, s_{c}, s_{d}$ and $s_{e}$ have been using it. We also assume that the SU $s_{a}$ newly starts using

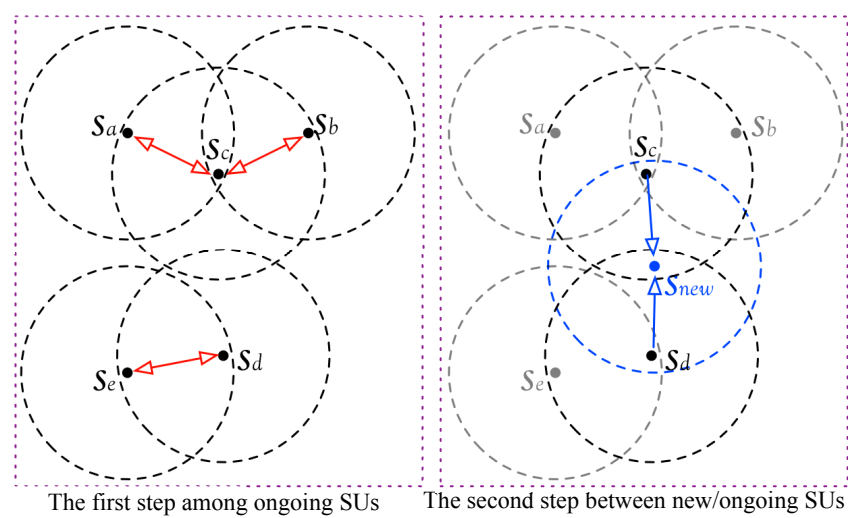

Fig. 4. Two-Step Information-Exchange method for SU transmitters.

channel $n$ in slot $h$. In this case, we call $s_{b}, s_{c}, s_{d}$ and $s_{e}$ as ongoing SUs on channel $n$, and call $s_{e}$ as new SU on channel $n$. Further, if each of the five SUs has the same subslot utilization threshold $\mathbb{U}=25 \%$, the new $s_{a}$ will interrupt the ongoing transmission of $s_{e}$ in slot $h$.

\section{B. Two-Step Information-Exchange}

To solve the above described competition problem, we suggest a simple method called Two-Step Information-Exchange (TSIE) for SUs. TSIE is accomplished by SUs during the second phase of every SU's slot. The process of doing TSIE is shown in Fig. 4.

The first step is performed by ongoing SUs among themselves via accessed channels. The information is about which available channels the ongoing SUs are using. Let $n_{m}(t)$ denote an available channel used by an ongoing SU $s_{m} \in S$ at time $t$. If no channel is used by $s_{m}$ at time $t, n_{m}(t)$ equals zero. By exchanging information with neighboring ongoing SUs via channel $n_{m}(t), s_{m}$ can obtain the number, denoted by $\psi_{m}^{n}(t)$, of SU competitors on channel $n_{m}(t)$. This is $\psi_{m}^{n}(t)=\sum_{i=1}^{M}\left[\xi_{m i}(t) \mid n_{i}(t)=n_{m}(t)\right]$.

The second step is initiated by new SUs and it is conducted between new and ongoing SUs. The information is about the competitor number perceived by different ongoing SUs. Consider that a newly arrived SU $s_{m^{\prime}} \in S$ wants to use channel $n$ in slot $h$. By communicating with a neighboring ongoing SU $s_{m}$, SU $s_{m}^{\prime}$ can get information of $\psi_{m}^{n}(t)$ from $s_{m}$. Similarly, after communicating with all neighboring ongoing SUs, $s_{m}^{\prime}$ can learn the largest number, denoted by $y_{m^{\prime}}^{n}(t)$, of SU competitions on channel $n$ as being:

$y_{m^{\prime}}^{n}(t)=\max \left\{\psi_{i}^{n}(t) \mid n_{i}(t)=n, \xi_{m^{\prime} i}(t)=1, i=1,2, . ., M\right\}$

where $y_{m^{\prime}}^{n}(t)$ is called competition level for $s_{m^{\prime}}$ to access channel $n$ at time $t$. If $\left(y_{m^{\prime}}^{n}(t)+1\right) \mathbb{U}$ is not larger than one, then SU $s_{m}^{\prime}$ can use channel $n$. Otherwise $s_{m}^{\prime}$ has to look for other available channels in order to protect ongoing SUs using channel $n$. We further let $T_{\text {com }}$ denote the maximum of SU competitors accommodated by the same channel. Then, we have $T_{\text {com }}=\lfloor 1 / \mathbb{U}\rfloor$. 
Clearly, for new SUs, the competition level on a channel indicates how heavily the channel is used by ongoing SUs. The larger the competition level on a channel is, the lower the channel availability becomes.

\section{Channel Selection}

So far, we have formulated two pairs of parameters, $\left(x^{n}, E_{\text {idle }}^{n}\right)$ and $\left(y_{m}^{n}, T_{\text {com }}\right)$. It is clear that these parameters vary in distinct metrics and measures. This gives rise to a two-constraint based decision problem of finding the most available channel for SUs. Fuzzy logic is suggested to solve this problem because of the capability of coping with various criteria for decision making purposes. We first introduce a parameter named "Fuzzy Channel Availability (FCA)".

\section{A. Fuzzy Channel Availability}

Proposition: Fuzzy Channel Availability is a fuzzy logic based parameter to represent three different levels of channel availability for SUs. The three levels are respectively formalized as three fuzzy sets, namely, "high-level", "medium-level" and "low-level" channel availabilities.

The use of FCA is to map different types of parameter values to an uniform type, i.e., fuzzy membership degree. Let $\sigma$ denote a parameter of either $x^{n}$ or $y_{m}^{n}$, i.e., $\sigma \in\left\{x^{n}, y_{m}^{n}\right\}$. The value of the parameter $\sigma$ at time $t$ is denoted by $\sigma(t)$. We use the notations $\alpha_{\sigma}, \beta_{\sigma}$ and $\gamma_{\sigma}$ to denote three fuzzy sets "highlevel", "medium-level" and "low-level" under parameter $\sigma$, respectively. Their membership functions are denoted by $g_{\sigma}^{\alpha}$, $g_{\sigma}^{\beta}$, and $g_{\sigma}^{\gamma}$, respectively. Then, $g_{\sigma}^{\alpha}(\sigma(t)), g_{\sigma}^{\beta}(\sigma(t)), g_{\sigma}^{\gamma}(\sigma(t)) \in$ $[0.0,1.0]$ are defined as membership degrees of $\sigma(t)$ to fuzzy sets $\alpha_{\sigma}, \beta_{\sigma}$, and $\gamma_{\sigma}$, respectively. The three membership degrees form a vector $V_{\sigma}(t)=\left(g_{\sigma}^{\alpha}(\sigma(t)), g_{\sigma}^{\beta}(\sigma(t)), g_{\sigma}^{\gamma}(\sigma(t))\right)$.

We call $V_{\sigma}(t)$ the FCA-based characterization of parameter $\sigma$ at time $t$. As $V_{\sigma}(t)$ is a three-coordinate vector, it is not convenient to carry out the numerical computing regarding decision making. This has prompted the development of methods to compound three coordinates into a joint value referred to the channel availability.

\section{B. Fuzzy-Comparison}

Towards the compounding goal, we adopt a fuzzycomparison based algorithm developed by Saaty [9]. The algorithm is based on using a paired-comparison of three fuzzy sets' importances in deciding on which channel is most available. Let $\pi_{\times}, \pi_{+}$, and $\pi_{-}$denote the importances of "high-level", "medium-level", and "low-level", respectively. For an example: i) since high-level has strong importance over low-level, we assign $\frac{\pi_{\times}}{\pi_{-}}$with 5 ; ii) since high-level (resp. medium-level) has weeker importance than mediumlevel (resp. low-level), we assign both $\frac{\pi_{\times}}{\pi_{+}}$and $\frac{\pi_{+}}{\pi_{-}}$with 3; iii) since high-level, medium-level, or low-level has equal importance over itself, we have $\frac{\pi_{\times}}{\pi_{\times}}=\frac{\pi_{+}}{\pi_{+}}=\frac{\pi_{-}}{\pi_{-}}=1$. We can therefore obtain a fuzzy-comparison matrix, denoted by $\Pi$, as:

$$
\Pi=\left[\begin{array}{lll}
\pi_{-} / \pi_{-} & \pi_{+} / \pi_{-} & \pi_{\times} / \pi_{-} \\
\pi_{-} / \pi_{+} & \pi_{+} / \pi_{+} & \pi_{\times} / \pi_{+} \\
\pi_{-} / \pi_{\times} & \pi_{+} / \pi_{\times} & \pi_{\times} / \pi_{\times}
\end{array}\right]=\left[\begin{array}{ccc}
1 & 3 & 5 \\
1 / 3 & 1 & 3 \\
1 / 5 & 1 / 3 & 1
\end{array}\right]
$$

The matrix $\Pi$ is used to determine the numerical values of $\pi_{\times}, \pi_{+}$, and $\pi_{-}$, respectively. Given the eigen value $\lambda$ and eigen vector $\Omega$ of matrix $\Pi$, they satisfy the eigen equation $\Pi \Omega=\lambda \Omega$ and characteristic equation $\operatorname{det}(\Pi-\lambda I)=0$, where $I$ is an unit matrix. The largest real eigen value corresponds to an eigen vector, denoted by $\Omega^{*}$ and given by $\Omega^{*}=\left\{\omega_{\times}, \omega_{+}, \omega_{-}\right\} \simeq\{0.94,0.31,0.19\}$. The three coordinates of vector $\Omega^{*}$ refer to values of $\pi_{\times}, \pi_{+}$and $\pi_{-}$, respectively.

Consequently, we compose three coordinates of $V_{\sigma}(t)$ in term of a linear combination:

$$
\xi_{\sigma}(t)=g_{\sigma}^{\alpha}(\sigma(t)) \omega_{\times}+g_{\sigma}^{\beta}(\sigma(t)) \omega_{+}+g_{\sigma}^{\gamma}(\sigma(t)) \omega_{-}
$$

where, $\xi_{\sigma}(t)$ is called the FCA-based decision factor of parameter $\sigma$ at time $t$ for channel selection. By using FCAbased decision factor, we develop the hybrid decision making (for channel selection) in the following subsection.

\section{Hybrid Decision Making}

Considering a channel $n$ available at time $t$, we first map the idle time statistics, i.e., the parameter pair $\left(x^{n}, E_{\text {idle }}^{n}\right)$, to fuzzy membership degree of $x^{n}$ to $g_{x^{n}}^{\alpha}, g_{x^{n}}^{\beta}$ and $g_{x^{n}}^{\gamma}$, respectively.

As an example, we choose values $0, E_{\text {idle }}^{n}$ and $2 E_{\text {idle }}^{n}$ to indicate that, under the three values, the availability of channel $n$ is exactly equivalent to high-level, medium-level and lowlevel, respectively, i.e., $g_{x^{n}}^{\alpha}(0)=g_{x^{n}}^{\beta}\left(E_{\text {idle }}^{k}\right)=g_{x^{n}}^{\gamma}\left(2 E_{\text {idle }}^{k}\right)=1.0$. But as described in subsection III-B, the availability of channel $n$ is assumed to decrease with $x^{n}(t)$. This implies that: $\mathrm{i}$ ) when $x^{n}(t)$ is increasing, the channel availability is far away from the high level and becomes closer to the low level, ii) when $x^{n}(t)$ is increasing and it is smaller than $E_{i d l e}^{k}$, the channel availability becomes closer to the medium level, and iii) when $x^{n}(t)$ is increasing and it is larger than $E_{i d l e}^{k}$, the channel availability is far away from the medium level. We therefore have:

- $g_{x^{n}}^{\alpha}\left(x^{n}(t)\right)$ should not increase with $x^{n}(t)$.

- $g_{x^{n}}^{\beta}\left(x^{n}(t)\right)$ should not decrease before $x^{n}(t)$ reaching $E_{\text {idle }}^{n}$ and not increase after $x^{n}(t)$ exceeding $E_{i d l e}^{n}$.

- $g_{x^{n}}^{\gamma}\left(x^{n}(t)\right)$ should not decrease with $x^{n}(t)$.

Thus, we formalize the membership functions of FCA under $x^{k}$ as simple linear functions:

$$
\begin{aligned}
& g_{x^{n}}^{\alpha}\left(x^{n}(t)\right)= \begin{cases}1-\frac{x^{n}(t)}{E_{\text {idle }}^{k}}, & 0 \leq x^{n}(t)<E_{\text {idle }}^{k} \\
0, & \text { others }\end{cases} \\
& g_{x^{n}}^{\beta}\left(x^{n}(t)\right)= \begin{cases}\frac{x^{n}(t)}{E_{\text {idle }}^{n},} & 0 \leq x^{n}(t)<E_{\text {idle }}^{n} \\
2-\frac{x^{n}(t)}{E_{\text {idle }}^{n}}, & E_{\text {idle }}^{k} \leq x^{n}(t)<2 E_{\text {idle }}^{n} \\
0, & \text { others }\end{cases} \\
& g_{x^{n}}^{\gamma}\left(x^{n}(t)\right)= \begin{cases}\frac{x^{k}(t)}{E_{\text {idle }}^{n}}-1, & E_{\text {idle }}^{k} \leq x^{n}(t)<2 E_{\text {idle }}^{n} \\
0, & \text { others }\end{cases}
\end{aligned}
$$

For the parameter pair $\left(y_{m}^{n}, T_{\text {com }}\right)$, we know that the larger $y_{m}^{n}(t)$ is, the lower the availability of channel $n$ for SU $s_{m}$ becomes. Therefore, we adopt similar membership functions 


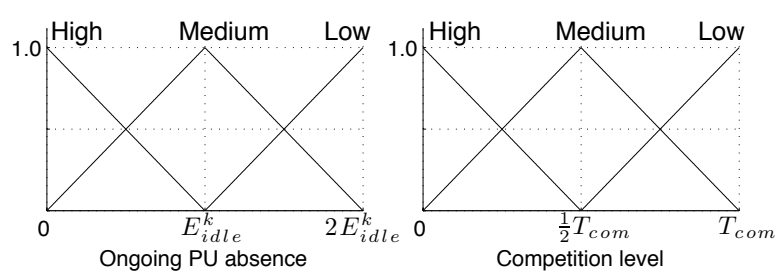

Fig. 5. Membership functions of $x^{n}(t)$ and $y_{m}^{n}(t)$ to FCA.

with regard to equations (5), (6) and (7). The difference is that we set $g_{y_{m}^{n}}^{\alpha}(0)=g_{y_{m}^{n}}^{\beta}\left(T_{\text {com }} / 2\right)=g_{y_{m}^{n}}^{\gamma}\left(T_{\text {com }}\right)=1.0$. We illustrate in Fig. 5 the membership functions under parameters $x^{n}$ and $y_{m}^{n}$, respectively. According to equation (4), the FCA-based decision factors of $x^{n}$ and $y_{m}^{n}$ at time $t$, denoted by $\xi_{x^{n}}(t)$ and $\xi_{y_{m}^{n}}(t)$ respectively, can be computed.

Although, the values of $\xi_{x^{n}}(t)$ and $\xi_{y_{m}^{n}}(t)$ have the same type with respect to FCA, their respective weights for decision making still need to be configured. Therefore, we introduce a variable $p_{r} \in[0.0,1.0]$, in which the decision maker configures $\xi_{x^{n}}(t)$ with weight $\left(1-p_{r}\right)$ and $\xi_{y_{m}^{n}}(t)$ with weight $p_{r}$. For a given SU $s_{m}$, the numerical channel availability of channel $n$ at time $t$ is finally given by:

$$
\theta_{m}^{n}(t)=\left(1-p_{r}\right) \xi_{x^{n}}(t)+p_{r} \xi_{y_{m}^{n}}(t)
$$

In the equation, $p_{r}$ is called hybrid coefficient of integrating both $\xi_{x^{n}}(t)$ and $\xi_{y_{m}^{n}}(t)$ when doing decision making. For instance, at $p_{r}=0$, the pure idle time based selection is performed. By computing the numerical channel availabilities of the channels of interest, the most available channel in this particular case is determined by the largest value of $\theta_{m}^{n}(t)$.

\section{Vi. Performance Evaluation}

In this section, we report the simulation results for performance evaluation of the suggested hybrid decision making algorithm. We simulated (in C++ language and using GNU Scientific Library [10]) a CR environment, where 100 SU transmitters are uniformly distributed over a $500 \mathrm{~m} \times 500 \mathrm{~m}$ district. The time periods of "PU presence" and "PU absence" are exponentially distributed with mean values $T_{p 1}$ and $T_{p 2}$, respectively. Every SU holds a time period $T_{s 1}$ before performing an access. The expected time period of a SU transmission is equal to a constant value $T_{s 2}$. Once an ongoing SU transmission is interrupted by PU's channel occupancy, the SU will restart transmission after a time interval equal to $T_{s 1}$ plus the remaining duration of $T_{s 2}$. The simulation parameter settings are presented in Table I.

\section{A. Simulation Scenarios and Performance Metrics}

To study how well the hybrid decision making affects the access behavior of SUs, we consider six scenarios: random based selection, pure idle time based selection, and hybrid decision making based selections with $p_{r}=0.1, p_{r}=0.2$, $p_{r}=0.4$ and $p_{r}=0.8$, respectively. The TSIE method is used in all six scenarios. The simulator runs in looping, and each loop stands for $10 \mathrm{~ms}$. Further, we run each scenario 40 times, and the simulation time of each run is 10000 s.
TABLE I

PARAMETER SETTINGS

\begin{tabular}{ll}
\hline Parameter & Value \\
\hline Sensing setting & $\delta=10 \mathrm{~ms} ; H=20000$ \\
Radio setting & $\mathbb{D}=200 m ; T_{\text {com }}=6$ \\
Number of channels & $N=10,15$ \\
Number of SUs & $M=100$ \\
SU activity & $T_{s 1}$, Uniform in $[1.0 s, 10.0 s] ;$ \\
& $T_{s 2}=1.0 s$ \\
PU activity & $T_{p 1}, T_{p 2}$, Uniform in $[1.0 s, 10.0 s] ;$ \\
& Idle time is beween $2.0 s$ and $20.0 s$ \\
\hline
\end{tabular}

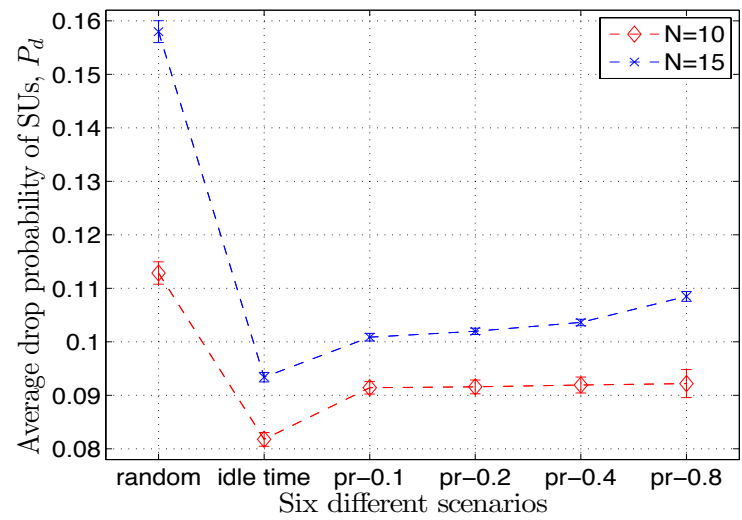

Fig. 6. Average drop probability of SUs versus six scenarios

Three metrics are used for performance evaluation, namely, average drop probability, average block probability and average success probability. They are denoted by $P_{d}, P_{b}$ and $P_{s}$, respectively. We define a drop as the event of a SU out from a channel due to the channel occupancy by PU. A block indicates a case when a SU is unable to access any channel as all of them are fully used by PUs and SUs. A success means that a SU transmitter successfully finishes the transmission once without interruption by PUs. Considering the $i^{\text {th }}$ simulation run of a scenario, assume that the $m^{t h} \mathrm{SU}$ performed $\alpha_{i, m}$ times of attempting channel access, while the SU got $\beta_{i, m}$ times of drops and $\gamma_{i, m}$ times of blocks. For 40 runs, we have:

$$
\begin{aligned}
& P_{d}=\frac{1}{40} \sum_{i=1}^{40}\left[\frac{1}{M} \cdot \frac{\sum_{m=1}^{M} \beta_{i, m}}{\sum_{m=1}^{M} \alpha_{i, m}}\right] \\
& P_{b}=\frac{1}{40} \sum_{i=1}^{40}\left[\frac{1}{M} \cdot \frac{\sum_{m=1}^{M} \gamma_{i, m}}{\sum_{m=1}^{M} \alpha_{i, m}}\right]
\end{aligned}
$$

and $P_{s}=1.0-P_{d}-P_{b}$.

\section{B. Results and Discussion}

The simulation results regarding the SUs' average drop and block probabilities are shown Figs. 6 and 7, respectively as well as the $95 \%$ confidence interval. The SUs' average success probability is shown in Fig 8 .

As observed in Fig. 6, for the case of fixed channel number $N$ (=10 or =15), the pure idle time scenario leads to the smallest dropping probability $P_{d}$, while $P_{d}$ in the random scenario is largest. The reason for this is that in pure idle time scenario the SUs have learned in advance the channel availability based on idle time statistics. Thus, the SUs may have more concentration on good channels, and the possibility 


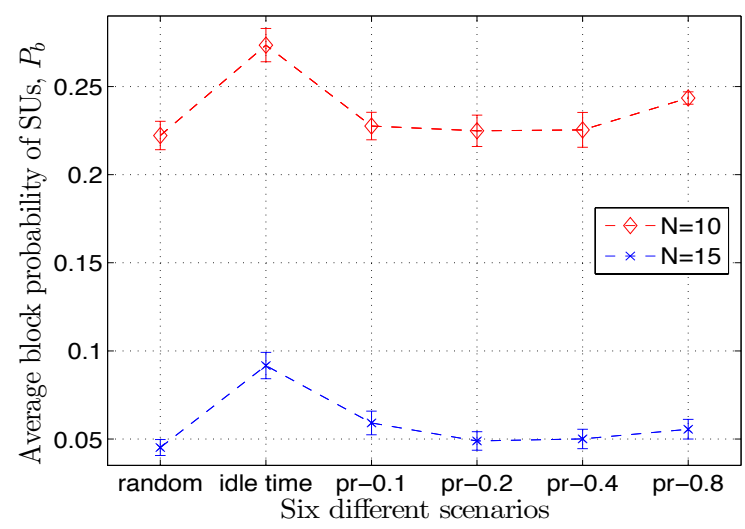

Fig. 7. Average block probability of SUs versus six scenarios

of being dropped due to PUs is reduced. Since in hybrid scenarios the SUs use statistical information in part, the corresponding values of $P_{d}$ under fixed $N$ are between the ones in random and pure idle time scenarios.

In Fig. 7, we observe that, under fixed $N$, the pure idle time scenario stands out as being the worst performing of the largest blocking probability $P_{b}$. This is because the limited channel capacity may make parts of SUs access the best channels, whereas other SUs are left with the worse channels. Fig. 7 further shows that, for the same scenario, $P_{b}$ under $N=15$ is smaller than the one under $N=10$. This is because of the larger number of channels provided for SUs under $N=15$ than under $N=10$. However, the possibility of SUs being dropped may increase with $N$ (i.e., from 10 to 15). Hence, for the same scenario, $P_{d}$ under $N=15$ is larger than the one under $N=10$, as shown in Fig. 6

To investigate the overall performance of different channel selection algorithms, we study the SU's average success probability $P_{s}$. In Fig. 8 , we observe that: i) under $N=15, P_{s}$ in pure idle time scenario is smaller the ones in four hybrid scenarios, and ii) under $N=10, P_{s}$ in pure idle time scenario is smallest. This means that: i) by learning only from idle time statistics, SUs are good at looking for the best channels, and ii) this may increase the number of SUs with the starvation of available channels. By using the hybrid decision making, every SU can learn how heavily the interested channels are used by other SUs (on average). Thus, the SUs are able to make a trade-off between the long remaining idle time and the low SU competition level when doing channel selection. In Fig. 8, the gain from trade-off is in the sense that, under $N=15$, $P_{s}$ in $p_{r}-0.2$ scenario is about $6.6 \%$ and $4.2 \%$ larger than the ones in random and pure idle time scenarios, respectively.

\section{CONCLUSION}

In this paper, a SU competition-based channel selection algorithm for cognitive radio networks has been developed. The idle time statistics based on information about PU channel occupancy have been derived. An information-exchange based method has been suggested for SUs to learn the SU competition level on available channels. From fuzzy logic point of

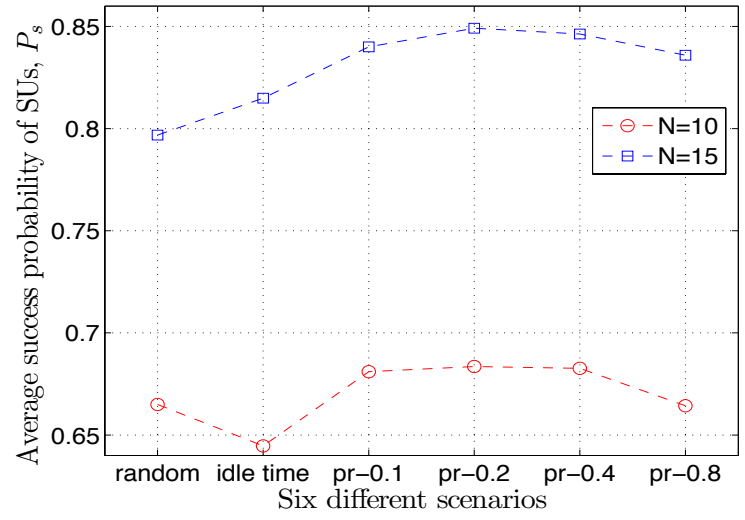

Fig. 8. Average success probability of SUs versus six scenarios

view, the idle time statistics and SUs' competition level have been integrated into a hybrid decision criterion. The channel selection has been optimized by the decision that the largest value referred to the hybrid decision criterion indicates the most available channel. Under suitable hybrid coefficient, simulation results have demonstrated that the overall performance of our developed algorithm outperforms both random and pure idle time based channel selection algorithms

\section{ACKNOWLEDGMENT}

The authors gratefully acknowledge the support of Euro-NF Network of Excellence for the work presented in this paper. The work was done as part of the Euro-NF project Networking over Cognitive Radio Networks (NETCO).

\section{REFERENCES}

[1] I.F. Akyildiz, W.-Y. Lee, M.C. Vuran, and S. Mohanty, "NeXt generation/dynamic spectrum access/cognitive radio wireless networks: a survey", Elsevier Computer Networks, vol. 50, pp. 2127-2159, 2006.

[2] Q. Zhao, L. Tong, A. Swami, and Y. Chen "Decentralized cognitive MAC for opportunistic spectrum access in ad hoc networks: a POMDP framework", IEEE Journal on Selected Areas in Communications, vol. 25, no. 3, pp. 589-600, April 2007.

[3] L. Yang, L. Cao, and H. Zheng, "Proactive channel access in dynamic spectrum networks", IEEE International Conference on Cognitive Radio Oriented Wireless Networks and Communications, Orlando, USA, Augest 2007.

[4] L. Akter, B. Natarajan, and C. Scoglio, "Spectrum usage modeling and forecasting in cognitive radio networks", Cognitive Radio Networks, pp. 37-60, Auerbach Publications, 2009.

[5] X. Zhang and H. Su, "Opportunistic spectrum sharing schemes for CDMA-based uplink MAC in cognitive radio networks", IEEE Journal on Selected Areas in Communications, vol. 29, no. 4, pp. 716-730, April 2011.

[6] A. Popescu, D. Erman, M. Fiedler, A. Popescu, and D. Kouvatsos, "A middleware framework for communication in cognitive radio networks", International Congress on Ultra Modern Telecommunications and Control Systems, Moscow, Russia, October 2010.

[7] R.R. Chen, K.H. Teo, and B. Farhang-Boroujeny, "Random access protocols for collaborative spectrum sensing in multi-band cognitive radio networks", IEEE Journal on Selected Areas in Communications, vol. 5, no. 1, pp. 124-136, February 2011.

[8] Y.H. Bae, A.S. Alfa, and B.D. Choi, "Performance analysis of modified IEEE 802.11-based cognitive radio networks", IEEE Communications Letters, vol. 14, no. 10, pp. 975-977, May 2009.

[9] T.L. Saaty, "Exploring the interface between hierarchies, multiplied objectives and fuzzy sets", Fuzzy Sets and Systems 1, pp.57-68, 1978.

[10] GSL - GNU Scientific Library, http://www.gnu.org/s/gsl/ 